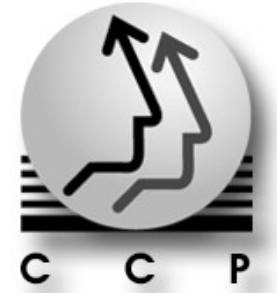

Población y Salud en Mesoamérica

Revista electrónica publicada por el

Centro Centroamericano de Población,

Universidad de Costa Rica, 2060 San José, Costa Rica

http://ccp.ucr.ac.cr

Población y Salud en Mesoamérica

Revista electrónica semestral, ISSN-1659-0201

Volumen 10, número 1, informe técnico 1

Julio - diciembre, 2012

Publicado 1 de julio, 2012

http://ccp.ucr.ac.cr/revista/

\title{
Influencia de las condiciones socio-demográficas en las actitudes $y$ el comportamiento en sexualidad que expresan los y las jóvenes de noveno año provenientes de un colegio público del cantón Central de Alajuela, Costa Rica
}

\author{
Élida Vargas-Barrantes \\ Carlomagno Araya-Alpízar
}

\section{(cc) BY-NC-ND}

Protegido bajo licencia Creative Commons

Centro Centroamericano de Población 


\title{
Influencia de las condiciones socio-demográficas en las actitudes y el comportamiento en sexualidad que expresan los y las jóvenes de noveno año provenientes de un colegio público del cantón Central de Alajuela, Costa Rica
}

\author{
Influence of socio-demographic conditions in attitudes and behavior in sexuality that \\ express the youth of ninth year from a public school in the Canton Central Alajuela, Costa \\ Rica
}

\section{RESUMEN}

Élida Vargas-Barrantes ${ }^{1}$,Carlomagno Araya-Alpízar ${ }^{2}$

Objetivo: analizar las condiciones socio-demográficas en jóvenes de noveno año provenientes de un colegio público del cantón Central de Alajuela, Costa Rica, para determinar cómo influyen estas condiciones en las actitudes y comportamiento en sexualidad que ellos expresan. Método: la recolección de la información se hizo por medio de un cuestionario, validado de previo con jóvenes de noveno año que provienen de un colegio público. Se seleccionó una muestra simple aleatoria de 33 estudiantes de un total de 181 activos hasta noviembre de 2011. El análisis de los datos cuantitativos se realizó mediante el programa SPSS 20.0 y Excel 2010. Resultados: se confirmó que los jóvenes prefieren a sus familiares cercanos como principal fuente para aclarar sus dudas sobre el tema de sexualidad. Además, se corroboró que con una edad promedio de 15,5 años, el $24 \%$ de los jóvenes han iniciado relaciones sexuales coitales. La mayoría de estos jóvenes usan las pastillas como método anticonceptivo. Conclusiones: los jóvenes están deseosos por aclarar sus dudas en el tema. Aún cuando ellos siguen apostando por sus padres como principal medio para hacer sus consultas, la televisión y los amigos en la calle representan opciones importantes en cuanto al tiempo que ellos dedican como pasatiempo. Por su parte, el inicio tan temprano de las relaciones sexuales íntimas se asocia con los altos índices de embarazos en adolescentes en América Latina. Como adultos, padres de familia y docentes, tenemos un enorme compromiso en proveer un ambiente más confiable y seguro a nuestros jóvenes.

Palabras clave: diagnóstico, educación sexual, comportamiento sexual, jóvenes, prácticas sexuales.

\begin{abstract}
Objective: analyze the socio-demographic conditions in ninth year youth from a public school of the Canton in Alajuela Central, Costa Rica, to determine how these conditions influence on attitudes and behavior in sexuality that they express. Method: the collection of information was through a validated questionnaire prior ninth year young people who come from a public school. Selected a simple random sample of 33 students from a total of 181 assets until November 2011. The quantitative data analysis was performed using SPSS 20.0 and Excel 2010 program. Results: it was confirmed that young people prefer their close relatives as their primary source to clarify their doubts on the subject of sexuality. In addition, was corroborated that with an average age of 15.5, 24 youth have initiated coital sex. Most of these young people use pills for contraception. Conclusions: young people are eager to clarify their doubts on the subject. Even though they are still betting on parents as primary means to make inquiries, television and friends on the street represent important options for the time they spend as a hobby. For its part, as early initiation of intimate intercourse is associated with high rates of pregnancies in adolescents in Latin America. As adults, parents and teachers, we have a huge commitment in providing a more reliable and secure our youth environment.
\end{abstract}

Keywords: diagnosis, sex education, sexual behavior, young people, sexual practices.

Recibido: 1 feb. 2012

Aprobado: 8 mar. 2012

\footnotetext{
${ }^{1}$ Universidad de Costa Rica, Sede de Occidente. COSTA RICA. elida.vargas@ucr.ac.cr

${ }^{2}$ Universidad de Costa Rica, Sede de Occidente. COSTA RICA. carlo.araya@ucr.ac.cr
} 


\section{INTRODUCCION}

Para Krauskopf (2003), la adolescencia es un período de exploración, experimentación con el sexo, de fantasías y realidades sexuales y de incorporación de la sexualidad en la identidad de la persona. Los adolescentes tienen una curiosidad casi insaciable hacia los misterios del sexo.

Hoy nadie pone en duda que toda educación sexual supone información sexual. De este modo, el niño y más tarde el adolescente serán capaces de decidir sobre lo que más les conviene para sus vidas, sin verse obligados a aceptar determinadas instancias o poderes que se les planteen. Esta etapa de decisión solo se puede alcanzar si se ha recibido un mínimo de información objetiva, porque la experiencia por sí sola, no es suficiente para superar los obstáculos que se pueden presentar en el aprendizaje de cuestiones relacionadas con la sexualidad (Muñoz-Cadavid et al., 2005).

La sexualidad es una construcción cultural $y$, por consiguiente, no se representa como una función biológica marcada por la idea de función/disfunción, natural/patológico, normal/anormal. Hablar sobre sexo con los niños, los adolescentes, los hombres o las mujeres constituye hoy una forma de controlar el comportamiento (Faccioli de Camargo y Ribeiro, 2003). En la opinión de Amaro (2005), la educación que se da en el hogar es muy importante y los establecimientos educativos solo complementan ese gran trabajo. No obstante, Castro et al., (2006), reconocen que en las labores educativas que se relacionan con la sexualidad, el desempeño del educador tiene momentos tensos, producto de numerosos prejuicios existentes en la cultura, de las contradicciones entre las funciones asignadas y las que está preparado para desempeñar.

El reto de los colegios y demás instituciones educativas es brindar todo el apoyo necesario a la familia para que pueda cumplir eficazmente sus funciones en la estimulación y fortalecimiento de los valores, siempre respetando los espacios familiares, los derechos y los deberes de los padres (Muñoz, 2010). Por su parte, Amaro (2005), enfatiza que en la actualidad, aunque muchos padres aprueban la enseñanza de educación sexual en las escuelas, en la práctica siempre han habido opiniones encontradas, ya que algunos de ellos rechazan la educación sexual argumentando razones religiosas y/o morales.

Para Polaino-Llorente (2006), la paradoja surge cuando los hijos llegan a la adolescencia porque los padres experimentan una gran ignorancia y no saben cómo comportarse con ellos en temas sobre sexualidad. Por eso, es fundamental el consenso acerca de lo que se dice y se practica tanto en casa como en el colegio a través de la educación y sus planes de estudio. En este sentido, la información brindada por los padres no cumple las expectativas de los niños y jóvenes, lo que ocasiona curiosidad y búsqueda de rápidos referentes a través de la Internet, de la televisión y los medios de comunicación de poca seguridad y credibilidad.

Asimismo, es evidente que la globalización ha influido en que los adolescentes se encuentren expuestos a influencias multiculturales. Los insumos tradicionales, como la televisión, la Internet, las imágenes publicitarias, entre otros, no son los únicos que recibe la juventud; su desarrollo ocurre junto a múltiples estímulos. Ello ha roto la homogeneidad de las culturas y, por consiguiente, la inmovilidad de los roles. De ahí que, la inclusión social permite el ejercicio de 
los derechos políticos, civiles y sociales, el acceso a programaciones con oportunidad para la incorporación social en el presente y el desarrollo de las posibilidades de calidad de vida (Krauskopf, 2003).

Sin embargo, la sociedad envía a los jóvenes mensajes contradictorios. Por ejemplo, se espera que los niños no tengan ningún interés por el sexo y que los adultos "normales" sean sexualmente activos dentro del matrimonio, pero no se proporcionan pautas claras acerca de cómo debería producirse la transición de un niño sin interés por el sexo a un adulto sexualmente activo. Se trata, entonces, de una de las transiciones vitales más importantes y, por lo tanto, merece mayor atención (Muñoz, 2010).

Por eso, ante las contradicciones que experimentan nuestros jóvenes, los riesgos a los que se ven expuestos y la falta de responsabilidad por ofrecerles información oportuna y pertinente, en este estudio se evalúa la influencia de las condiciones socio-demográficas en las actitudes y comportamiento en sexualidad que expresan los jóvenes de noveno año provenientes de un colegio público del cantón Central de Alajuela, Costa Rica.

\section{MÉTODO Y DATOS}

\subsection{Características del grupo de estudio}

Para el presente estudio se seleccionó un colegio público del cantón Central de Alajuela. Este centro de enseñanza se clasifica con un tipo de Dirección 2. En esta categoría, el Ministerio de Educación Pública (MEP) incluye a los colegios que tienen matriculados entre 751 y 1500 estudiantes.

El estudio se realizó en el mes de noviembre del 2011, es decir, a finales del ciclo lectivo y se contó con la participación de dos grupos elegidos al azar para un total de 33 estudiantes. Según la última estadística del mes de octubre de ese año, la matrícula en noveno año en dicha institución estaba constituida por 78 hombres (43\%) y 103 mujeres (57\%) para un total de 181 estudiantes.

Se seleccionaron estudiantes de noveno año, ya que después de la revisión de algunos programas de III y IV ciclo se pudo constatar que los contenidos sobre sexualidad se ubican básicamente, y de manera directa, en materias que corresponden al III Ciclo. También, se analizaron y compararon las dos propuestas de ejes transversales del MEP vigentes desde el 2004 y el abordaje del tema de sexualidad como parte integral del currículo transversal.

Por lo anterior, la evaluación se realizó en esas fechas, con el propósito de lograr que los jóvenes hubieran estudiado los contenidos correspondientes al tema sexualidad humana, según lo que se propone el Ministerio de Educación Pública para las diversas materias que se estudian en el III ciclo. 


\subsection{El instrumento de evaluación}

Para el grupo seleccionado, en el presente estudio, se elaboró y se ajustó un instrumento de evaluación (cuestionario) con base en condiciones socio-demográficas que influyen en las actitudes y en el comportamiento en sexualidad que expresan los jóvenes en el III ciclo de Educación Básica. Para ello, el cuestionario primero fue evaluado en un grupo de noveno año, de un colegio público del cantón Central de Grecia. El instrumento consta de una motivación y 16 preguntas para marcar con equis " $\mathrm{x}$ ".

El análisis de datos cuantitativos se realizó mediante el programa SPSS 20.0 y Excel 2010, y el estudio descriptivo se hizo con la finalidad de recoger, clasificar, resumir y analizar las características de la población. Posteriormente, se realizaron algunas correlaciones entre variables.

\section{RESULTADOS}

En cuanto a la distribución por sexo para los estudiantes de secundaria que comprende el estudio, un total de 19 mujeres (58\%) y 14 varones (42\%) respondieron el cuestionario. Cuando se evaluó la edad de los estudiantes de noveno año, los datos confirmaron los resultados esperados para una población de adolescentes que estudian en un colegio diurno; de modo que, las mujeres tienen en promedio 15,5 años, y no existe diferencia significativa en los promedios de edad entre hombres y mujeres.

Por lo que toca a la constitución de las familias de donde provienen los adolescentes, el 91,3\% proviene de hogares constituidos por ambos padres y sus hijos. Son pocos quienes viven con el padrastro, la madrastra o algún otro miembro no identificado.

Con respecto a la creencia religiosa, el $61 \%$ de los estudiantes encuestados indicó profesar la fe católica, un $15 \%$ correspondió a los evangélicos y los cristianos respectivamente, y menos de un $10 \%$ de los entrevistados no indicó preferencia religiosa.

En relación con la ocupación del tiempo libre, se observaron diferencias muy importantes en los hábitos según sexo (Ver Figura N. ${ }^{\circ}$ 1). De acuerdo con los resultados, las actividades para ocupar el tiempo libre fueron, en su mayoría, dominadas por mujeres. Por ejemplo, del total de jóvenes que tienen el hábito de estudiar, el $62 \%$ son mujeres. Al analizar otras actividades recreativas que prefieren los estudiantes, las opciones "escuchar música" y "ver televisión" resultaron ser las alternativas más seleccionadas, con un $94 \%$ y un $79 \%$ respectivamente.

Además, la actividad "leer" representa una ocupación seleccionada por solo 6 de los 33 estudiantes, de los cuales cinco son mujeres. Por su parte, los hombres predominan en la práctica del deporte, es decir, que del total de estudiantes que practican una actividad deportiva, el 58\% corresponde a hombres.

De igual forma, cuando los estudiantes tienen alguna duda o inquietud en aspectos relacionados con la sexualidad, en primer lugar, el 58\% lo conversan con su pareja, padres, hermanos, 
hermanas u otros parientes (Ver Figura $\mathrm{N}^{\mathrm{o}}$ 2); en segundo, un $33 \%$ de los adolescentes encuentra la respuesta a las preguntas relacionadas con la sexualidad en la calle, con sus amigos o amigas y, en tercer lugar, un $27 \%$ de los jóvenes buscan alternativas a sus inquietudes en los programas de televisión. Es importante señalar que fueron muy pocos quienes indicaron buscar ayuda con los sacerdotes o consejeros espirituales.

Asimismo, y como se aprecia en la Figura $\mathrm{N}^{\mathrm{o}} 3$, el 24\% de los jóvenes de noveno año, han tenido relaciones sexuales. La edad promedio para el inicio de las relaciones coitales es de 15 años y no se presentan diferencias significativas entre hombres y mujeres. Entre ellos, el 15\% utilizan las pastillas anticonceptivas como método para prevenir un posible embarazo, mientras que el $9 \%$ restante utiliza el condón.

Todos los jóvenes manifestaron mantener la primera relación con el novio o novia y en un 50\% de los casos, la frecuencia de las relaciones las refieren como "de vez en cuando", es decir, una vez al mes. La decisión de tener relaciones íntimas fue por mutuo acuerdo para la mayoría de las parejas, aunque un $25 \%$ de los jóvenes manifestaron que las relaciones sexuales se dieron bajo presión por parte de la pareja.

\section{DISCUSIÓN}

Por las características de la población, la diferencia de 10 unidades porcentuales entre el número de hombres y mujeres es proporcional a la variación entre sexos que se mantiene para los 181 estudiantes que constituyen la población de noveno hasta finales de noviembre del 2011.

La sexualidad humana es todavía un tema cargado de prejuicios y tabúes, por esto, y en la mayoría de los casos, el tema es omitido o tratado de manera bastante inadecuada en la escuela y en la familia (Faccioli de Camargo y Ribeiro, 2003). A estos aspectos hay que sumarles el hecho de que existe una tendencia en los jóvenes a iniciar las relaciones sexuales a edades cada vez más tempranas.

En el estudio de Fernández et al. (2000), los investigadores encontraron que la edad promedio para la primera relación sexual coital fue mayor en mujeres que en hombres. Para estos autores, el deseo sexual y la curiosidad aparecen como los principales motivos que llevaron a los encuestados a tener esta experiencia (González-Garza, et al., 2005). También coinciden en que sobresale una mayor actividad sexual en aquellos jóvenes que viven en localidades urbanas en comparación con quienes viven en localidades rurales.

En efecto, encuestas demográficas y de salud realizadas con mujeres en Centro y Sur América señalan que altos porcentajes de adolescentes en México, Guatemala, Ecuador, El Salvador y Brasil tuvieron sus primeras relaciones sexuales antes de los 15 años (Moreno et al., 2006).

De igual forma, el estudio de Romero et al., (2008), con jóvenes limeños, demostró que el uso del preservativo en las primeras relaciones fue escaso debido a que estas no fueron planeadas o simplemente por el deseo de experimentar una primera relación sin condón, lo cual incrementa las relaciones de riesgo. 
Para el caso de Costa Rica, la Encuesta Nacional sobre Sexualidad Adolescente del Ministerio de Salud, realizada en el 2010 y que abarcó a casi 2.000 estudiantes de todo el país, reveló un inicio de la actividad sexual más prematuro del que se pensaba (Ministerio de Salud, 2011). Algo más del $23 \%$ de los encuestados respondió que tuvo su primera relación sexual antes de los 14 años.

Frente a estos datos, el inicio más temprano de la actividad sexual encontrada en la Encuesta Nacional coincide con los resultados encontrados por los autores de este estudio, ya que el 24\% de los jóvenes de noveno del colegio seleccionado de Alajuela, con una edad promedio de 15,5 años, indicó haber tenido relaciones sexuales íntimas.

Dicha encuesta también mostró que ante la pregunta "tuvo relaciones sexuales durante el último año" y si en ese lapso de tiempo "usó condón", las respuestas indicaron que a menor edad es menor también el cuidado, ya que entre los jóvenes de 13 años, sólo un 39\% respondió que sí usó preservativo. La cifra creció al $43 \%$ en los adolescentes de 14 , y un poco más, hasta alcanzar el $47 \%$ entre los de 15 años. Sin embargo, en todos los casos se trata de menos de la mitad del universo de los encuestados (Ministerio de Salud, 2011). En este estudio, los autores llegaron a resultados aún más inquietantes, menos del 38\% de los jóvenes indicaron usar el condón como método anticonceptivo, mientras que para el resto fue suficiente con usar pastillas anticonceptivas.

Según el Ministerio de Salud (2011), en la combinación del no uso del condón con el inicio de la actividad sexual a edad temprana, la conclusión es obvia: la mayoría de estudiantes del nivel medio se encuentran desprotegidos ante la eventualidad de un contagio de enfermedades de trasmisión sexual y expuestos a embarazos adolescentes, cada vez más comunes. Así es, los adolescentes se convierten en un grupo vulnerable frente a las enfermedades de transmisión sexual porque practican acciones que involucran peligro, arriesgando su bienestar con consecuencias negativas para la salud (Marquez, 2006).

Las relaciones sexuales en edades tan precoces, los escasos conocimientos sobre sexualidad y la sobre valoración de dichos conocimientos por parte de los propios alumnos, transforma a los jóvenes (principalmente a los de estratos socioeconómicos más bajos) en grupos de riesgo, por lo que se hace necesario intervenir con adecuados planes de educación sexual, prevención de enfermedades de transmisión sexual y embarazos precoces no deseados (Fernández et al.; 2000).

Al respecto, Torriente et al. (2010), también argumentan que las causas para el inicio temprano de las relaciones sexuales son, entre otras, la precocidad del desarrollo sexual, la pobre educación sexual en cuanto a salud sexual y reproductiva que poseen por diferentes causas, así como características educacionales, religiosas, sociales, culturales, económicas y psicológicas. Además, en cuanto a la labor de los padres en la educación sexual de sus hijos, según estos últimos autores, los padres son una fuente de afecto, simpatía y comprensión, un lugar para la experimentación y con los que, efectivamente, se debe contar para la transmisión de determinados conocimientos.

Para lograr la madurez sexual, se requiere además de la madurez biológica, tener experiencias apropiadas consigo mismo, así como una comunicación sincera y directa con el padre, la madre o la persona cuidadora, desde los primeros años de vida. La sexualidad humana, señala Mock 
(2005), aunque tiene base biológica, es mayormente aprendida; por eso, es necesario que los padres adopten un papel más activo al discutir temas de sexo con sus hijos para ayudar a darles respuestas a dudas que ellos tengan, siempre hablando con la verdad, para lo cual su consentimiento en temas de sexo es indispensable

Por su parte, la comunicación debe llevarse a cabo en una atmósfera de respeto y deseo de comprensión. La importancia en la comunicación entre progenitores y sus hijos, se confirma en un estudio de Pérez et al., (2005), quienes, a partir de una muestra integrada por 250 adolescentes de décimo año con una deficiente comunicación padres-hijos, encontraron que, para los adolescentes, la escuela se convirtió en el fundamento de la información sexual.

Ya propiamente en el trabajo con los estudiantes en el aula, Ramírez (2000), señala que, con el propósito de cubrir los temas de sexualidad que marca el programa educativo, el profesor recurre constantemente a otros profesionales fuera de la escuela o del salón de clases. A los psicólogos, las enfermeras, los médicos y los trabajadores sociales entre otros, se les otorga una responsabilidad que en realidad le corresponde al docente. Si los instructores no cuentan con la preparación adecuada, no se puede esperar que los alumnos adquieran de ellos los conocimientos oportunos sobre el tema. Además, es necesario un clima de confianza donde se demuestre a los jóvenes que pueden acercarse con familiaridad a conversar con el docente cada vez que lo requieran y no sólo en aquellos momentos en que se cuenta con la visita de otro profesional.

Aún en el tercer milenio, la educación sexual en niños, niñas y adolescentes continua padeciendo graves deficiencias al mantener un carácter sexista, estandarizada, despersonalizada y cargada de mitos y tabúes (Lescaille, 2009). Para Chaves (2003), es evidente que la cultura moderna hace una separación entre persona y sexualidad para relacionar esta última como algo que es indiferente a la persona que la ejerce, para transformar la sexualidad en un objeto que se puede comerciar.

Los autores también encontraron en el estudio que para los jóvenes de noveno, los sacerdotes o guías espirituales no representan una opción para aclarar dudas en torno a la sexualidad. Se espera que la Iglesia, por medio de los guías espirituales, represente un espacio de conversación e información alterno a la familia, a los pares, a los medios de comunicación y a los mismos docentes. Sin embargo, las religiones también parten de abordajes automatizados y generalizados de la sexualidad, sin valorar que la capacidad de respuesta sexual es diferente para cada individuo según su desarrollo y condiciones que le rodean, así como la educación recibida y experiencias previas.

Claro está que esta es una forma de evadir el tema, que no sólo se presenta a nivel de religiones o creencias religiosas. Para Faccioli de Camargo y Ribeiro (2003), posibilitar espacios para que los niños hablen sobre la sexualidad humana y para manifestar su sexualidad rompe patrones arraigados en los modelos existentes que consisten en proponer referencias ideales que dificultan contactar con el mundo de manera creativa y singular. Carballo (2002), también considera que es de vital importancia conocer y reflexionar sobre la capacidad de respuesta sexual humana para evitar comportamientos erróneos y llenos de culpa, los cuales pueden llevar al deterioro progresivo de las habilidades emocionales. 
Los medios de comunicación también hacen un aporte significativo al inicio precoz de las relaciones sexuales en jóvenes. Claramente se evidencia que en la Internet, la escena virtual expone la fantasía y se deja vía libre a la imaginación (Escalante-Romero et al., 2008). Para estos autores, el medio permite establecer relaciones sin ningún tipo de censura, siempre y cuando sean toleradas por aquellos que las comparten. Es indudable que este desplazamiento de funciones educativas debiera ser asumida por la familia, sustentada, en alguna medida, en la crítica a la televisión y más recientemente a la Internet, que llegan a colocarse como responsables de malestares familiares y sociales (Díaz, 2008).

En este momento, tampoco se puede obviar la relación antagónica entre la comunicación familiar y tiempo excesivo dedicado a observar la televisión. Al respecto, Díaz (2008), indica claramente que en la televisión $\mathrm{y}$ en otros medios es frecuente encontrar información que, desafortunadamente, obvian madres, padres y docentes, dentro de la cual ocupa un lugar considerable lo relacionado con la sexualidad.

En concordancia con los resultados obtenidos en nuestra investigación, Escalante-Romero et al. (2008), encontraron en su estudio que el primer compañero sexual de los alumnos, fue en la mayoría de los casos, su propio novio o novia. Para González et al., (2007), los jóvenes tienen relaciones sexuales generalmente con sus parejas, en especial las mujeres, mientras que el sexo ocasional ocurre más en los hombres. En su estudio con adolescentes de Educación Media, estos autores encontraron que, en promedio, el 89,1\% del total de adolescentes inició la actividad sexual con su pareja. No obstante, al separar los sexos encontraron diferencias significativas, por ejemplo, las mujeres representaron el $90,3 \%$ y los hombres el 79,4\%, es decir, los varones mantienen en mayor proporción relaciones íntimas "oportunistas".

Desde la visión de Pérez y Pick (2006), contar con la habilidad de comunicación asertiva en la pareja sexual regular, aumenta la probabilidad de que los adolescentes se protejan. Dado que la comunicación asertiva es un factor clave para lograr la conducta sexual protegida, los programas de promoción de salud sexual deberían considerar la formación de esta habilidad para los adolescentes que atienden.

Finalmente, los resultados encontrados en el estudio nos llevan a enfatizar sobre la propuesta del Ministerio de Salud (2010), en la necesidad de ampliar y profundizar la educación sexual en las escuelas de Costa Rica. Niños y adolescentes no pueden seguir expuestos a información imprecisa, descontextualizada y mercantilista. Los adultos -como padres, educadores o amigos de ellos- estamos obligados a ofrecerles un ambiente más seguro, pero, sobre todo, la información que les permita tomar decisiones acertadas y sin prejuicios. 


\section{CONCLUSIONES}

- La temprana edad de inicio de las relaciones sexuales genitales impone una clara necesidad de establecer programas y servicios de educación sexual integral (dentro y fuera del sistema escolar) que inicie en edades previas a la adolescencia. Acompañando estos esfuerzos, deben fortalecerse los servicios de salud desde una perspectiva de amabilidad, oportunidad y calidad que aseguren el acceso a la información y a métodos de protección y anticoncepción (Ministerio de Salud, 2011).

- La adolescencia es una etapa de la vida definitoria en muchos aspectos, también puede ser decisiva en cuanto a la opción entre estilos de vida saludables, que garanticen un futuro más protegido de la enfermedad, y otros riesgos que implican graves peligros para el mantenimiento de la salud y de la vida misma (Morlachetti, 2007). En este caso, cualquier descuido u olvido en el uso de los condones representa una opción potencial para adquirir enfermedades y un riesgo para la vida.

- Cada niño tiene que aprender actitudes, conocimientos y destrezas, con vistas a vivir su sexualidad de modo auto determinado y enriquecedor (Lemos, 2007). Lejos de representar un riesgo para incentivar o motivar las relaciones sexuales íntimas, la educación sexual es el mejor medio de prevención que, a la vez, aumenta la autoestima y, sobre todo, retarda el inicio de la actividad sexual en los jóvenes.

- La educación comienza desde el nacimiento y la familia tiene un lugar primordial en ella, los padres son los primeros y principales educadores sobre la sexualidad de los hijos. La familia es el primer lugar donde la persona realiza la experiencia del amor. Donde encuentra modelos de mujer y de varón de la relación entre ambos; por lo tanto, su papel en la maduración de la sexualidad es básico e insustituible. De ahí que la preparación permanente de todos y cada uno de los miembros de la familia se convierta en un reto impostergable.

- En la mayoría de las escuelas, los profesores a menudo no permiten espacio para la discusión sobre sexualidad, no hay una acción dialógica franca y abierta sobre las ansiedades y preocupaciones sexuales por el temor de no saber responder con eficacia las preguntas de los adolescentes y el temor de despertar el deseo sexual en ellos (Rodríguez, 2010). En este sentido, el docente también está llamado a la formación permanente, pero primero debe buscar superar sus limitaciones y ansiedades. Como en cualquier otra materia, el docente no tiene por qué saberlo todo, pero sí se requiere de disposición personal y comprensión hacia la diversidad.

- La educación debe ser en sí un proceso de interacción entre la comprensión y la explicación del mundo social y natural y ser capaz de conducir al ser humano a altos niveles de bienestar espiritual y material (Megna, 2011). Por eso, resulta preocupante que después de varios años de formación en primaria y secundaria, los jóvenes no logren superar aspectos que por tantos años se han manejado como un tabú. 
- Esto implica que la formación científica que se espera de la Educación Básica no ha logrado superar las grandes imprecisiones con que maneja el tema en los medios populares. La sexualidad, por su alto grado de estigmatización social, sigue siendo aún objeto de demasiados mitos que han perdurado en el tiempo debido, entre otros, al escaso interés que ha suscitado este tema entre las ramas de la ciencia implicadas. No obstante, gracias al avance científico, el error se ha puesto en evidencia y es momento de actuar para cambiar los resultados. 


\section{BIBLIOGRAFÍA}

Amaro, M. (2005). Sexualidad y bioética. Rev Cubana Med. Gen. Integr. 21 (1-2): 1-11.

Chaves, M. (2003). Perspectiva sexual de los adolescentes. Revista Inter Sedes IV(7): 95-107.

Carballo, S (2002). Educación de la expresión de la sexualidad humana. Educación. 26(1):29-46.

Castro, P.; Torres, M.; García, R. (2006). Las necesidades de educación sexual de los jóvenes estudiantes. La Habana: Ministerio de Educación/UNFPA.

Díaz, C. (2008). Sexualidad y televisión infantil: huellas de doble vínculo. Rev. Sexología y Sociedad. 14(38): 1-15.

Escalante-Romero, L.; Cerrón-Vela, C.; Salazar-Granara, A.; Mezones-Holguín, E. (2008). Descripción de La Conducta Sexual En Adultos Jóvenes Limeños. Revista Horizonte Médico. 8(1): 73-80

Faccioli de Camargo, A.; Ribeiro, C. (2003). La educación sexual en lo cotidiano de la escuela. Educar 31: 67-85.

Fernández, L.; Bustos, L.; González, L.; Palma, D.; Villagrán, J.; Muñoz, S. (2000). Beliefs and attitudes towards sexual education among adolescents aged 11 to 17 years old. Rev. Méd.Chile. 128(6):8-17

González, E.; Molina, T.; Montero, A.; Martínez, V.; Leyton, C (2007). Comportamientos sexuales y diferencias de género en adolescentes usuarios de un sistema público de salud universitario. Rev. Méd. Chile.135: 1261-1269

González-Garza, C.; Rojas-Martínez, M.; Hernández-Serrato, M.; Olaiz-Fernández, G. (2005). Perfil del comportamiento sexual en adolescentes mexicanos de 12 a 19 años de edad. Resultados de la ENSA 2000. Salud Pública de México. 47(3): 1-15

Krauskopf, D. (2003). Participación social y desarrollo en la adolescencia. Costa Rica: Fondo de Población de las Naciones Unidas.

Lemos, A. (2007). Resumen de Entre lo dicho y no dicho sobre sexualidad en la formación de la/el enfermera/o. Enfermería global. 6(10):1-14.

Lescaille, B. (2009). Evaluation of sexuality in adolescence from the sexual stereotypes. Medisan. 13(2):1-5

Márquez, M. (2006). Autoeficacia en comunicación sobre temas de sexo de padres a hijos. México: Universidad Autónoma de Nuevo León. Facultad de enfermería. Maestría en Ciencias de Enfermería. Trabajo final de graduación. 
Megna, A. (2011). La educación de la sexualidad: una mirada desde una perspectiva actual a las necesidades de los/las adolescentes y jóvenes. Cuba: Universidad de Las Tunas. Trabajo final de graduación para optar por el grado de Profesor de Física, Pedagogía y Didáctica de la Educación Superior.

Ministerio de Salud. (2011). Encuesta de Salud Sexual y Reproductiva 2010. San José, Costa Rica: El Ministerio.

Mock, G. (2005). Una mirada a la sexualidad: del nacimiento a la pubertad. Revista de Ciencias Sociales. 14:22-40.

Moreno, M.; León, M.; Becerra, L. (2006). Conducta sexual, conocimiento sobre embarazo y necesidades percibidas con relación a educación sexual, en adolescentes escolarizados. Espacio Abierto. 15 (4): 787-803.

Morlachetti. A. (2007). Políticas de salud sexual y reproductiva dirigidas a adolescentes y jóvenes: un enfoque fundado en los derechos humanos. CEPAL. Notas de Población. 85: $1-15$

Muñoz, M. (2010). Diagnóstico y propuesta metodológica de ejes temáticos para la educación San Pedro Pascual de la ciudad de Ibarra, provincia de Imbabura. México: Universidad Tecnológica América. Instituto de Investigación y Posgrados. Maestría en Educación.

Muñoz-Cadavid, M.; Revenga-Sánchez, M. (2005). Aprendizaje y educación afectivo-sexual: una revisión de los planteamientos iniciales del aprendizaje de las cuestiones sexuales. Revista Galego-Portuguesa de Psicoloxía e Educación. 10(12): 1

Pérez, C.; Pick, S. (2006). Conducta Sexual Protegida en Adolescentes Mexicanos. Revista Interamericana de Psicología. 40 (3): 333-340

Pérez, M.; Torres, J.; Peña, M.; Ramírez, T.; Pría, M. (2005). Conocimientos y comportamientos sobre salud sexual y reproductiva. Rev. Cubana Enfermería. 21 (1):13-24

Polaino-Lorente, (2006). Educación de los sentimientos y la sexualidad. Revista Española de Pedagogía. LXIV(325): 429-452.

Ramírez, T. (2000). Conocimientos y actitudes hacia la sexualidad en profesores de educación secundaria. Revista Sonorense de Psicología. 13(2): 47-54.

Rodríguez, M. (2010). Talleres educativos en sexualidad del adolescente: la escuela como escenario. Enfermería Global. 20:1-8.

Torriente, N.; Caballero, D. Rizo, A.; Menéndez; L. (2010). Conocimientos elementales sobre educación sexual en alumnos de una escuela secundaria básica urbana. Revista Habanera de Ciencias Médicas. 9(4) 576-587. 
Figura 1. Actividades en que ocupan el tiempo libre los estudiantes según sexo. Estudiantes de noveno año provenientes de un colegio público de Alajuela, Dirección Dos (noviembre 2011).

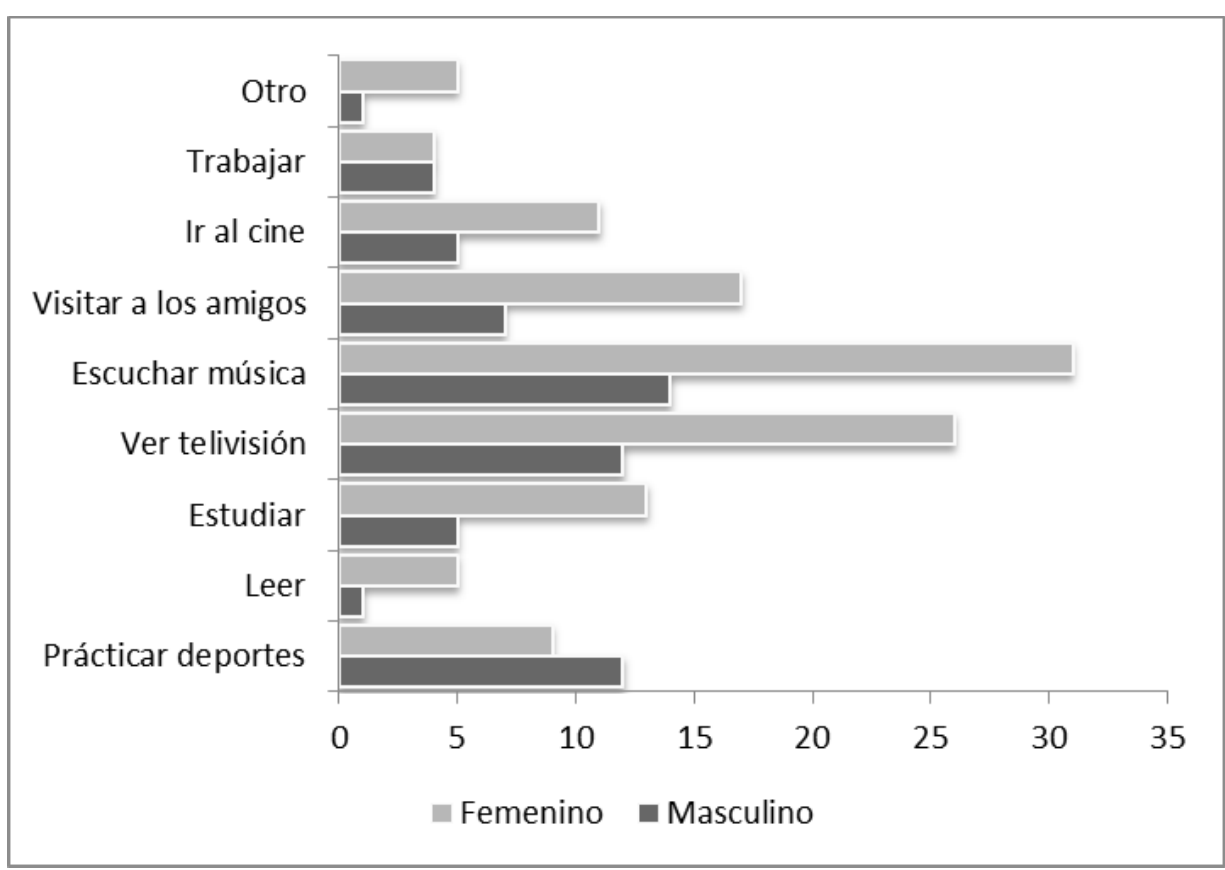

Figura 2. Fuente de consulta que prefieren los estudiantes cuando tiene alguna duda o inquietud en aspectos relacionados con la sexualidad. Estudiantes de noveno año provenientes de un colegio público de Alajuela, Dirección Dos (noviembre 2011).

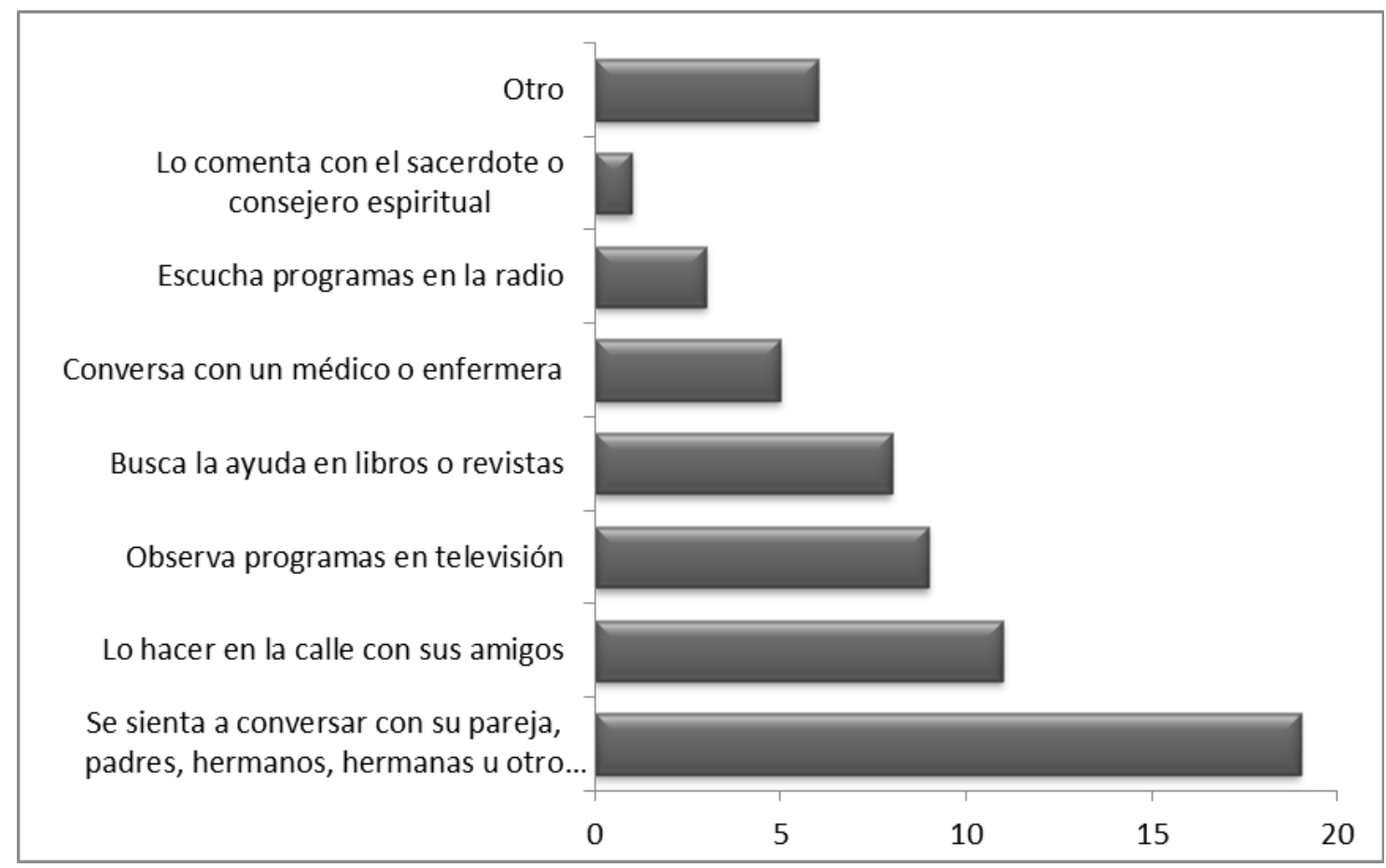


Figura 3. Cantidad de estudiantes que han tenido relaciones sexuales íntimas y los métodos anticonceptivos empelados. Estudiantes de noveno año provenientes de un colegio público de Alajuela, Dirección Dos (noviembre 2011).

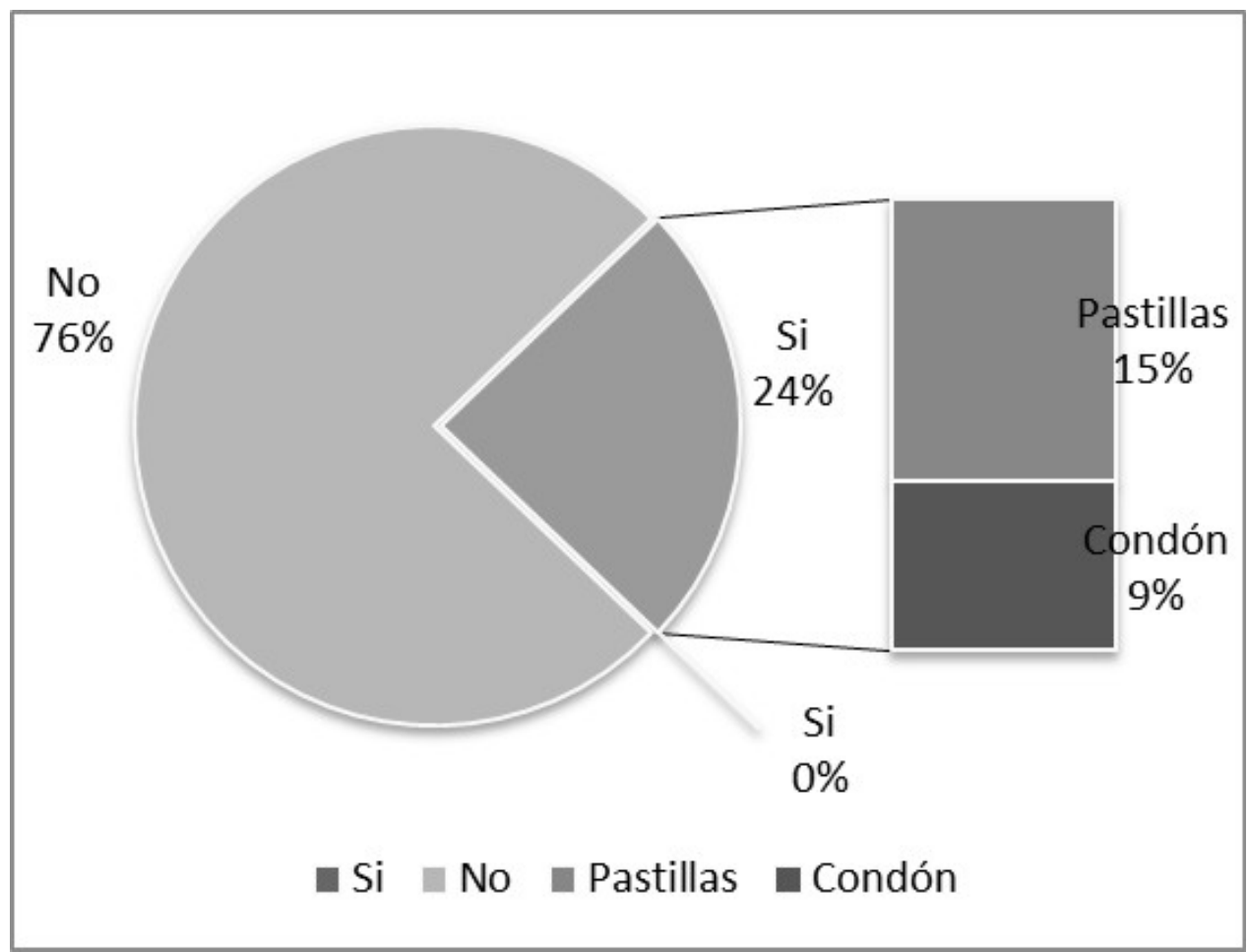

